\title{
A METHOD TO CHARACTERIZE THE INFLUENCE OF AIR DISTRIBUTION ON THE COMPOSTING TREATMENT: MONITORING OF THE THERMAL FIELDS
}

\author{
F. HENON ${ }^{1,2}$ \\ A. TREMIER ${ }^{1,2, *}$ \\ G. DEBENEST 3,4 \\ J-L. MARTEL ${ }^{5}$ \\ M. QUINTARD ${ }^{3,4}$
}

\author{
${ }^{1}$ Cemagref UR GERE 17 avenue de Cucillé \\ CS 64427, F - 35044 Rennes Cedex \\ ${ }^{2}$ Université Européenne de Bretagne, F-35000 Rennes, France \\ ${ }^{3}$ Université de Toulouse; UPS, INPT \\ Institut de Mécanique des Fluides de Toulouse \\ Allée du Prof. Camille Soula, 31400 Toulouse, France \\ ${ }^{4}$ CNRS; UMR 5502; IMFT \\ ${ }^{5}$ Suez Environnement, CIRSEE, \\ 38 rue du président Wilson 78230 Le Pecq France
}

Received: 23/09/08

Accepted: 05/02/09 *to whom all correspondence should be addressed: e-mail: anne.tremier@cemagref.fr

\begin{abstract}
In a composting process the monitoring of heat flows is a useful tool in terms of phenomenological comprehension and diagnosis of the process. Indeed, the temperature fields are generic markers of the micro-biological phenomena governing a composting process. Moreover, as heat flows are largely influenced by the air flowing through the porous solid waste, the temperature fields also characterize the influence of the air flow distribution on the composting process. However, at the industrial scale, it may be difficult to measure precisely the temperature distribution. In this paper, an in situ experimental data acquisition method of these thermal fields is described. Obtained data were used to reconstruct the evolution of the temperature in the whole composting reactor along the industrial composting process, in order to understand the influence of the air flow distribution. On the basis of these results, a model for the description of the thermal behaviour and the optimization of the aeration system was proposed.
\end{abstract}

KEYWORDS: composting, temperature distribution, modelling.

\section{INTRODUCTION}

One of the main differences between existing real scale and pilot scale composting processes concerns the characteristics of the gas flows that they allow. The gas flow patterns have a large influence on heat and mass transfers, i.e., on oxygen supply, moisture and temperature distribution within the waste (Haug, 1993; Tremier et al., 2005a). As a consequence, they can have a high impact on the quality of the end-product (kinetics of biodegradation, stage of stabilization and hygienization of the compost) but also on the environmental impact of the treatment (gaseous emissions and odours).

In order to better understand and optimize composting processes, the gas flow has to be characterized at a real scale and its influence on the composting process has to be modelled. In this objective, an experimental method has to be developed in order to characterize the gas flow through the porous waste mass all along the composting treatment.

The monitoring of the temperature field, considered as the simplest mean to provide the rate and extent of composting (Tiquia et al., 1996a; 1996b; 1997), can be used to analyse the performance of the aeration during composting processes. Indeed, temperature variation during composting is a good indicator of the process performance (NRAES, 1992) due to the close relationship between heat production and micro-organisms behaviours. During composting, temperature rises to thermophilic range due to heat generated by aerobic 
microbial decomposition of the organic waste and then decreases when the biological activity decreases along with the disappearance of the biodegradable organic matter. Furthermore, the evolution of the temperature is largely influenced by the air flow that supplies oxygen to the microorganisms and also exports heat and water.

However, studies of the temperature distribution were mostly set up at pilot scale (Fernandes et al., 1994; Kulcu and Yaldiz, 2008). Thus, the objective of this study was to develop an in situ characterization method of the gas flow behaviour all along the treatment. This method relied on the measurement of the evolution of the temperature distribution and the comparison with numerical modelling. Indeed, the temperature spatial variation within the waste volume was used to visualize the local aeration conditions while the global temperature variation along the composting period was used to analyse the performance of the process. Because temperature is an indirect indicator, understanding the results in terms of gas flow conditions required model validation. Thus a numerical model was developed and phenomenological assumptions were validated by the good agreement between experimental and numerical data.

\section{METHODS}

\subsection{Process and waste}

The studied composting process was led in a closed rectangular concrete reactor with positive forced aeration.

The treated waste was a mixture of sewage sludge and bulking agent (mixing ratio $1 / 3 \mathrm{~V} / \mathrm{V}$ ). The experimental dewatered sludge ( $13.4 \%$ of dry matter) was obtained from an activated sludge reactor treating the wastewaters of a slaughterhouse. The bulking agent was composed of green wastes recycled from a commercial plant composting slaughterhouse sludge. It took 5 days to prepare enough mixture to fill the reactor. Along its preparation the mixture was not submitted to forced aeration.

A $145 \mathrm{~m}^{3}$ heap of this mixture was loaded in the reactor and composted during four weeks without turning or new mixing. One may note however that the regular process operated on the industrial site consists of at least one turning in order to optimise sanitation and constancy of the end product composition.

Air was continuously blown from the bottom of the composting reactor, via two ventilation veins. The air flow was maintained to a quite constant flow rate, $\mathrm{Q}$, until four days before the end of the composting cycle. At this date, the flow rate was increased to $2.5 \mathrm{Q}$. Above the waste heap air was extracted and led to a biofiltration treatment system.

After four weeks, the composted mixture was unloaded and sieved on a rotating $20 \mathrm{~mm}$ grid, in order to separate the compost and the bulking agent.

\subsection{Data processing methodology}

During loading and unloading the waste mixture was weighed and samples were analysed for dry matter content, organic matter content, carbon and nitrogen content.

The evolution of the temperature distribution was measured by placing one hundred temperature sensors in the waste medium (during the loading of the reactor) at precise locations, following the network represented in Figure 1. This network was composed of eleven transversal sections (denoted from $A$ to $K$ ) each containing nine sensors (denoted from 1 to 9). The first three transversal sections and the last three ones were placed at a distance twice the one of the six sections in the centre of the composting reactor. This positioning of the temperature probes allows monitoring the temperature evolution in the transversal and longitudinal sections. Figure 1 presents a scheme of a transversal section of the composting reactor equipped with nine temperature sensors.

The temperature sensors registered data at regular time steps all along the composting cycle. The sensors were recovered and data analysed when the compost was sieved at the end of the four weeks. Obtained data allowed reconstructing the variation of the temperature distribution into the waste. To obtain these fields, day-average temperatures were calculated for each recovered sensor. Then, the temperature was linearly interpolated between each experimental point, using Matlab ${ }^{\mathrm{TM}}$. At points where the sensors could not be recovered, the temperature was also estimated by a linear interpolation from the temperatures of available neighbouring sensors. 

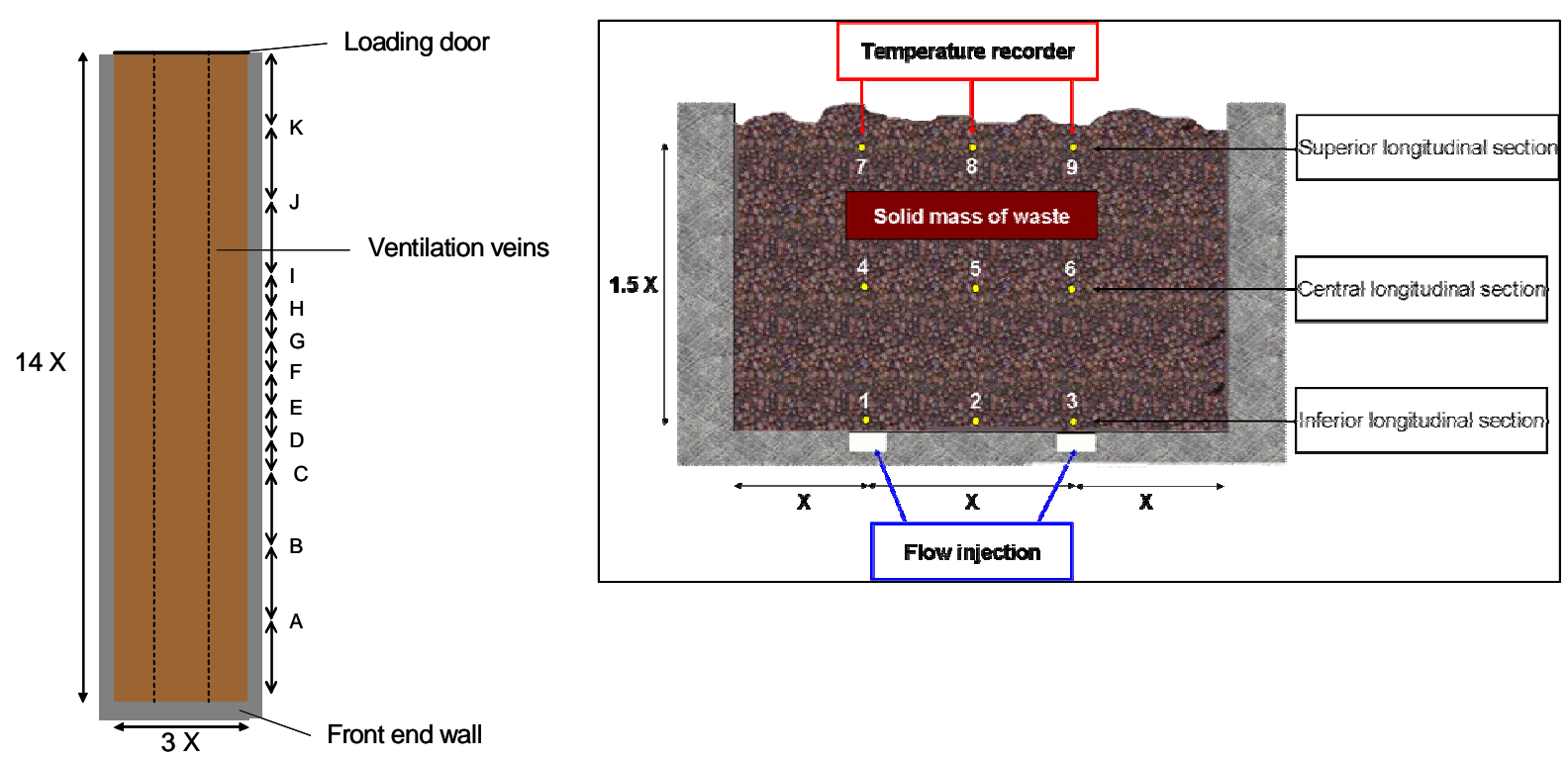

Figure 1. Longitudinal and transversal sections of the reactor equiped with temperature sensors

\section{RESULTS AND DISCUSSION}

\subsection{Composting mass balance}

Chemical characterizations performed on the mixture samples at the loading of the reactor and after four weeks composting allowed establishing mass balances of the composting process (Table 1). These balances traduce the performance of the treatment in terms of biodegradation.

Composting led to a significant mass loss (38.9\% of the initial mass) principally due to water loss. Indeed, temperature and air flow induced water transfer during the composting process . Biodegradation induced organic matter losses to the extent of $12.3 \%$ of the initial mass of organic matter. One may note that this biodegradation rate corresponds only to the degradation intervening within the composting reactor with forced aeration. Nevertheless, a certain amount of the mixtures had probably already begun to degrade during the preparation period.

Table 1. Variation of the mixture characteristics and mass balances

\begin{tabular}{ccccccc}
\hline & Time $=\mathbf{0}$ & \multicolumn{2}{c}{ Time $=\mathbf{4}$ weeks } & $\begin{array}{c}\text { Mass } \\
\text { balance }\end{array}$ & Loss \\
\cline { 2 - 7 } & $\begin{array}{c}\text { Concentration } \\
(\%)\end{array}$ & $\begin{array}{c}\text { Mass } \\
(\mathrm{T})\end{array}$ & $\begin{array}{c}\text { Concentration } \\
(\%)\end{array}$ & $\begin{array}{c}\text { Mass } \\
(\mathrm{T})\end{array}$ & $\mathrm{T}$ & $\begin{array}{c}\text { \% of the } \\
\text { initial mass }\end{array}$ \\
\hline Total mass & - & 97.12 & - & 59.36 & 37.76 & 38.9 \\
\hline Dry matter & 54.0 & 52.44 & 79.0 & 46.89 & 5.55 & 10.58 \\
\hline Water & 46.0 & 44.68 & 21.0 & 12.47 & 32.21 & 72.10 \\
\hline Organic matter & 92.0 & 48.25 & 90.2 & 42.30 & 5.95 & 12.33 \\
\hline Total carbon & 49.7 & 26.07 & 47.2 & 22.13 & 3.94 & 15.11 \\
\hline Total nitrogen & 2.5 & 1.30 & 2.7 & 1.27 & 0.03 & 2.31 \\
\hline
\end{tabular}

\subsection{Recovery rate of the temperature sensors}

About $70 \%$ of the temperature sensors were recovered at the end of the experiments, allowing a reasonable representation of the temperature fields on the transversal and longitudinal axis of the waste volume. It must be emphasized that this relatively high percentage of sensor recovery was achieved without disturbing the industrial process. Table 2 shows the number of recovered sensors for each transversal section. 
Table 2. Number of temperature sensors recovered for each transversal section

\begin{tabular}{cccccccccccc}
\hline $\begin{array}{c}\text { Transversal } \\
\text { section }\end{array}$ & A & B & C & D & E & F & G & H & I & J & K \\
\hline $\begin{array}{c}\text { Number of } \\
\text { sensors }\end{array}$ & $4 / 9$ & $7 / 9$ & $5 / 9$ & $8 / 9$ & $7 / 9$ & $9 / 9$ & $7 / 9$ & $6 / 9$ & $5 / 9$ & $7 / 9$ & $4 / 9$ \\
\hline
\end{tabular}

Transversal section $\mathrm{F}$ is a reference section because all temperature sensors were recovered during the screening operation. In addition, this section is located in the central part of the bin. Its temperature field is representative of the thermal behaviour without any boundary influence, especially heat losses at the extremities of the reactor, i.e., the front end wall or the loading door. Sections with the largest deficits in temperature sensors were sections A and K. Section $\mathrm{A}$ is close to the bottom wall and section $\mathrm{K}$ close to the loading door.

\subsection{Temperature distribution in longitudinal sections}

Figures 2, 3 and 4 present the variations of the temperature fields in the inferior, central and superior longitudinal sections. The locations of the sensors recovered for each section are represented on the initial temperature fields of each figure. Arrows indicate the location of the aeration veins. The colorbar is presented in degrees Celsius, and is specific to each figure.

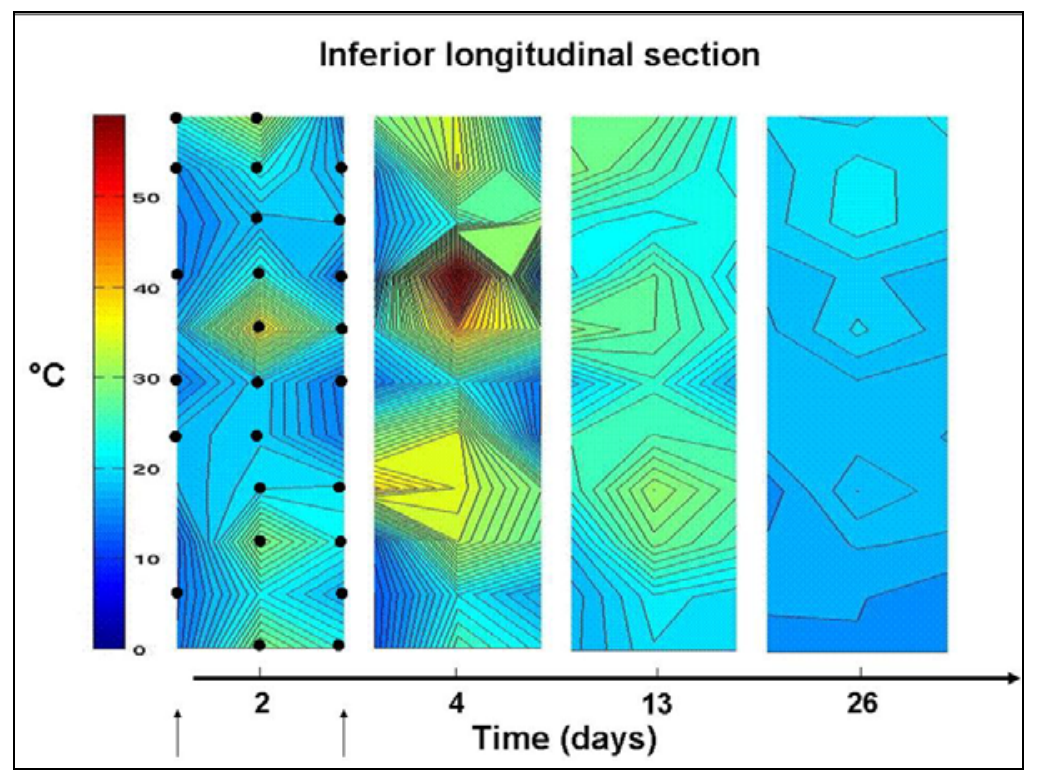

Figure 2. Variation of the temperature field in the inferior longitudinal section during the composting process

Temperature at the bottom of the composting reactor (Figure 2) remained cool all along the composting cycle, especially along the ventilation veins. Indeed, this area was largely influenced by the air injected into the substrate. Nevertheless, between the two ventilation veins, some points reached temperatures higher than $50^{\circ} \mathrm{C}$. At these locations, the temperature was characterized by a rapid increase during the first days (day 2 and 4 on figure 2) and then a slow decline until the end of the cycle. Such hot points indicate that the microbiological activity established at these locations and that the biological heat production was higher than the heat loss due to the air flow. Thus it traduces a lower influence of the forced aeration in-between the two ventilation veins at the bottom of the reactor. Moreover, the establishment of the microbiological activity, as characterized by the hot spots, proves that the oxygen supply was sufficient at these locations. The oxygen was probably supplied more through molecular diffusion phenomena than through forced convection phenomena. The presence of these heterogeneities in the temperature field may be linked to the heterogeneities in the physical properties of the solid substrate and especially to permeability differences. Indeed, the less permeable areas may be less influenced by cold air coming out of the ventilation veins, since they offer greater resistance to the flow. 
In the central longitudinal section (Figure 3), the temperature increased during the first few days with a maximum around $73^{\circ} \mathrm{C}$. This maximum is in agreement with the organic matter biodegradation shown by the mass balance. After this maximum, temperature dropped slowly until the end of the cycle. That drop is a consequence of the slowing of the biological activity. The temperature heterogeneities observed in the mid-cycle may be either due to local changes in physical properties of the substrate induced by the settlement, or to the location variation of some temperature probes that might also have been affected by the settlement. By the end of the cycle, temperature dropped to less than $20^{\circ} \mathrm{C}$ in the entire section. This means that biological heat was no longer produced at the bottom of the reactor. Stopping of the biological activity might be due to a lack of biodegradable organic matter or to an excess drying of the waste. This latter assumption is the most probable as aeration flow was increased few days before the unloading of the reactor. It is also confirmed by the high water loss (72\%) measured through the mass balance.

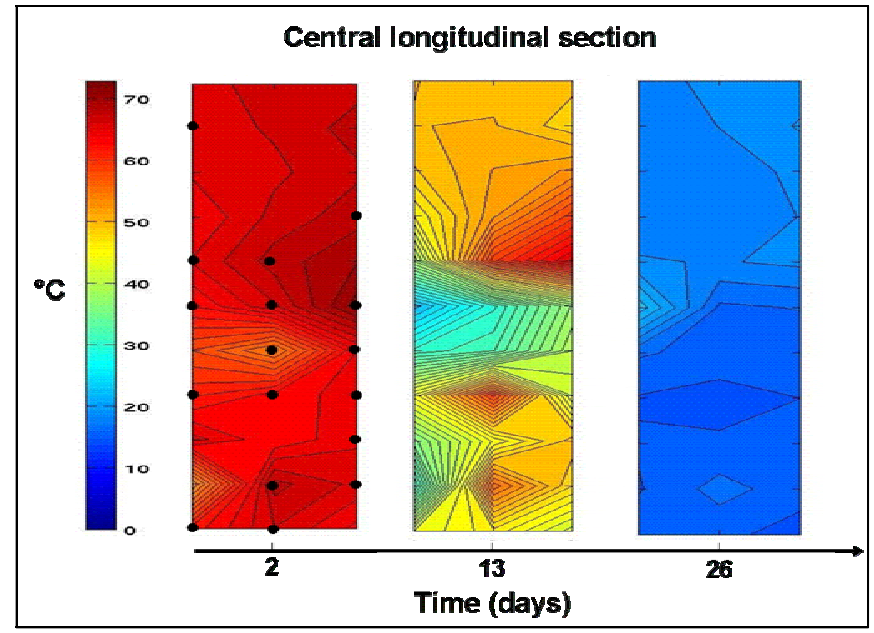

Figure 3. Evolution of the temperature field in the central longitudinal section during the composting process.

The variation of the temperature distribution (Figure 4) in the upper longitudinal section was very similar to the one obtained in the central longitudinal section. However, a cooler zone appeared throughout the cycle, close to the loading trap (top of Figure 4). This cooler zone was probably generated by the bevel shape of the waste heap at this location. This shape seems to facilitate heat transfer through the door constituted of a plastic tarpaulin. At the end of the cycle, temperature was higher preferentially in the central part of the reactor length, where the influence of heat loss due to the front end wall and the loading door was no longer significant.

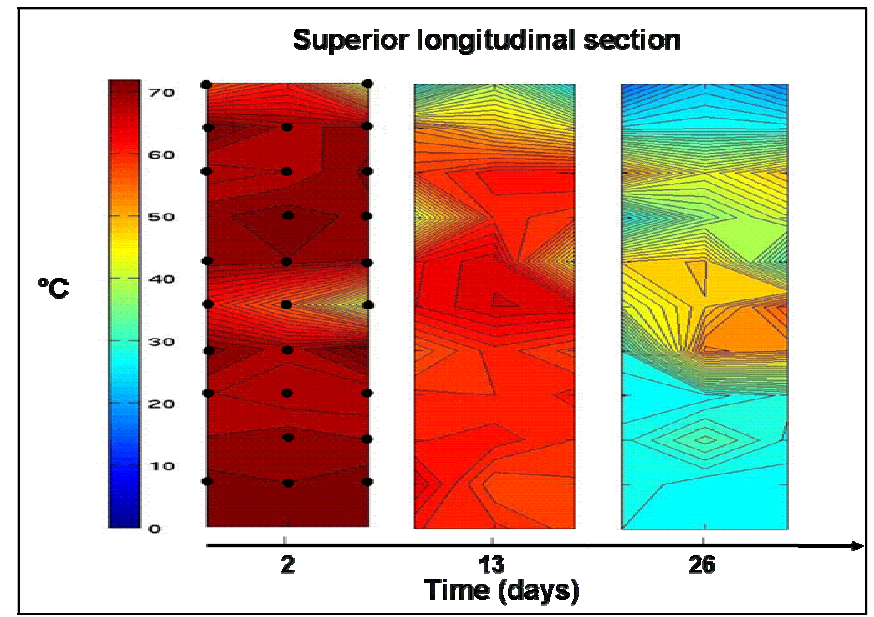

Figure 4. Evolution of the temperature field in the superior longitudinal section during the composting process 
The temperature levels differed from one longitudinal section to the other. These differences can be linked to the influence of aeration on the biodegradation condition, i.e., moisture and temperature, that control the biological activity (Richard, 2004).

Considering one longitudinal section at a given time, temperature can be considered as homogeneous along the composting reactor length (if not considering locations close to the loading door and the front end wall). The qualitative homogeneity of the thermal dynamics over the bin length seems to indicate, in a first approximation, that the temperature gradients in the bin longitudinal direction are small and that only the transverse temperature gradients are predominant. Moreover, the temperature distribution can be considered as symmetrical along the longitudinal plane of symmetry of the composting reactor.

On the basis of these results, the study of the variation of the temperature distribution was focused on the central transversal section $\mathrm{F}$

\subsection{Temperature distribution in the central transversal section $F$}

Figure 5 presents the variation of the temperature distribution in the transversal section $\mathrm{F}$ along the composting treatment (2,13 and 26 days composting). The locations of the temperature probes are represented on the initial temperature field. Arrows represent the location of the ventilation veins.

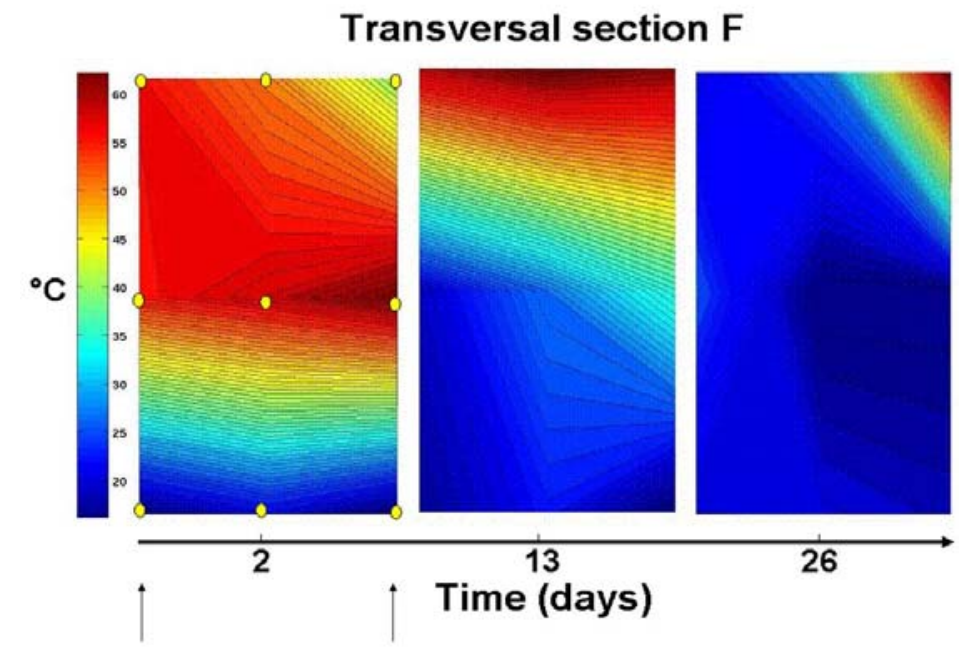

Figure 5. Evolution of the temperature field in the transversal section $\mathrm{F}$ during the composting process (arrows indicate location of injectors)

From the beginning of the composting cycle, temperature was high (above $50^{\circ} \mathrm{C}$ ) especially in the central and upper part of the sections. This temperature is characteristic of an already installed micro-biological activity in those areas. As already mentioned, the mixture of sludge and bulking agent was prepared and stored a few days before being loaded in the composting reactor and the biological degradation already began. However, near the ventilation veins, temperature was close to the temperature of the airflow injected into the waste, i.e., below $25^{\circ} \mathrm{C}$. In these areas, heat loss through the airflow was thus higher than the biological heat production. One may assume that the aeration not only cooled the porous medium (air temperature around $20^{\circ} \mathrm{C}$ ) but also dried it. Low temperature and lack of moisture might have reduced the biological activity. The strong influence of the airflow on heat transfers seemed to be restricted to a semi-spherical area around the ventilation veins, which is consistent with the streamlines distribution near the injection points.

In mid-cycle composting, the warm front moved in the upper part of the substrate. Indeed, air injected into the waste gradually got warmer and carried moisture during its ascent through the solid porous substrate. Thus, the optimum conditions for the biodegradation, in terms of temperature and moisture, preferentially moved from the bottom to the top of the reactor. Heat produced by biodegradation in the central part of the porous massif might also have heated the upper part by thermal convection. In the central part, an overall decline in temperature was observed. This temperature decline is characteristic of the slowing down of 
the biological activity observed after the temperature peak in a composting cycle. Indeed, one may assume that the major part of the easily biodegradable organic matter was consumed during the early stages of the composting cycle. Afterwards, the remaining organic matter was probably less accessible and more difficult to biodegrade. These assumptions would have to be verified in the future thanks to analytical characterization of the organic matter all along the composting treatment.

At the end of the cycle, the warm front has practically disappeared, except in a small area in the upper right corner of the reactor, where microbiological activity seems to persist. In the rest of the section, the temperature felt below $35^{\circ} \mathrm{C}$. This temperature fall in almost the entire reactor is probably amplified by a rapid drying of the solid substrate, imposed by an increase in the airflow rate few days before the end of the composting cycle.

The experimental data obtained on longitudinal and transversal sections showed that the aeration system and the airflow clearly influenced the composting behaviour and especially the thermal dynamics. The aeration system tends to cool the base of the reactor especially above the ventilation veins. In the rest of the reactor, heat transfer is mainly driven by convective flow. On an operational point of view, this means that the turning of the mixture, generally practised during a current composting operation, is necessary to ensure homogeneous biodegradation conditions (temperature and moisture). A modification of the airflow distribution at the bottom with a homogenization of the flow on the entire reactor bottom surface could also improve biodegradation. Other optimization ways, like temperature increase of the airflow entering the porous medium or variations of the air flow rate have also to be further investigated.

\subsection{Numerical results versus experimental results}

In order to validate the assumptions made on the basis of the experimental data and to test the ways of optimization of the composting process, a model has been developed.

As mentioned earlier the relative homogeneity of the thermal behaviour over the reactor length suggested that the thermal dynamics in a transverse section far from the front end wall and from the loading trap was representative of the thermal behaviour of the investigated process. Thus, a first 2D numerical model solving transport equations over a transverse section of the compost domain has been implemented using the software Comsol Multiphysics ${ }^{\circledR}$.

\section{Theorical hypothesis}

The modelling approach is based on the upscaling theory of transport in porous media and the following assumptions concerning the physical phenomena were made:

- The solid mass of waste is considered as a two-phase porous medium: a flowing gaseous phase and a static solid phase, to which an aqueous film containing the micro-organisms is tied. All transfer phenomena take place at the interface between the gaseous phase and the aqueous film.

- Thermal equilibrium between solid and gaseous phase is assumed.

- Convective and dispersive flows are considered

Biological reactions terms (organic matter degradation, oxygen consumption and water and heat production) were based on the model developed by Tremier et al. (2005b). Heat and mass transfers were modelled.

As an illustration of the model capabilities, equation for the energy balance is given below:

$$
\begin{aligned}
& \varepsilon \sum_{i \in g} C p_{g i} \frac{\partial \rho_{g} \omega_{g i} T}{\partial t}+(1-\varepsilon)\left[C p_{s H_{2} \mathrm{O}} \frac{\partial \rho_{s} \omega_{s \mathrm{H}_{2} \mathrm{O}} T}{\partial t}+C p_{s M S} \frac{\partial \rho_{s} \omega_{s M S} T}{\partial t}\right] \\
& =-\bar{\nabla} \cdot\left(\varepsilon \sum_{i \in g} C p_{g i} \rho_{g} \omega_{g i} T \bar{u}_{g}\right)+\bar{\nabla} \cdot\left(\overline{\bar{\Lambda}}_{\text {eff }} \cdot \bar{\nabla} T\right)+\Delta H_{\mathrm{O}_{2}} R_{\mathrm{O}_{2}}-\Delta H_{\mathrm{H}_{2} \mathrm{O}} \dot{m}_{g s \mathrm{H}_{2} \mathrm{O}},
\end{aligned}
$$

where the left hand side represents heat accumulation and the right hand side traduces respectively convective heat transfer, conductive heat transfer, heat production and heat loss through water vaporization. 
Comparison of experimental and numerical results

Figure 6 compares the temperature distribution obtained numerically after six days of composting and the corresponding experimental result of the reference section $F$.

The numerical profile obtained after six days of composting is highly comparable with its experimental counterpart. Indeed, the influence of the aeration veins in the lower part of the massif is clearly visible on both profiles. This influence is characterized by two quasihemispherical cool areas whose dimensions are essentially governed by the physical conditions of the substrate (permeability) and by the longitudinal and transverse dispersion of the gas in the volume (tortuosity, longitudinal and transversal dispersivities).

Moreover, the warm front on both profiles is concentrated in the middle and upper part where the microbiological activity is apparently well established.

Although many parameters, directly linked to the microbiological kinetics and having a large role on heat and mass transfers, have not been set in the numerical model (as variation of porosity, variation of permeability, etc.), the numerical results confirmed the assumptions on air flow behaviour and impacts on temperature profile.

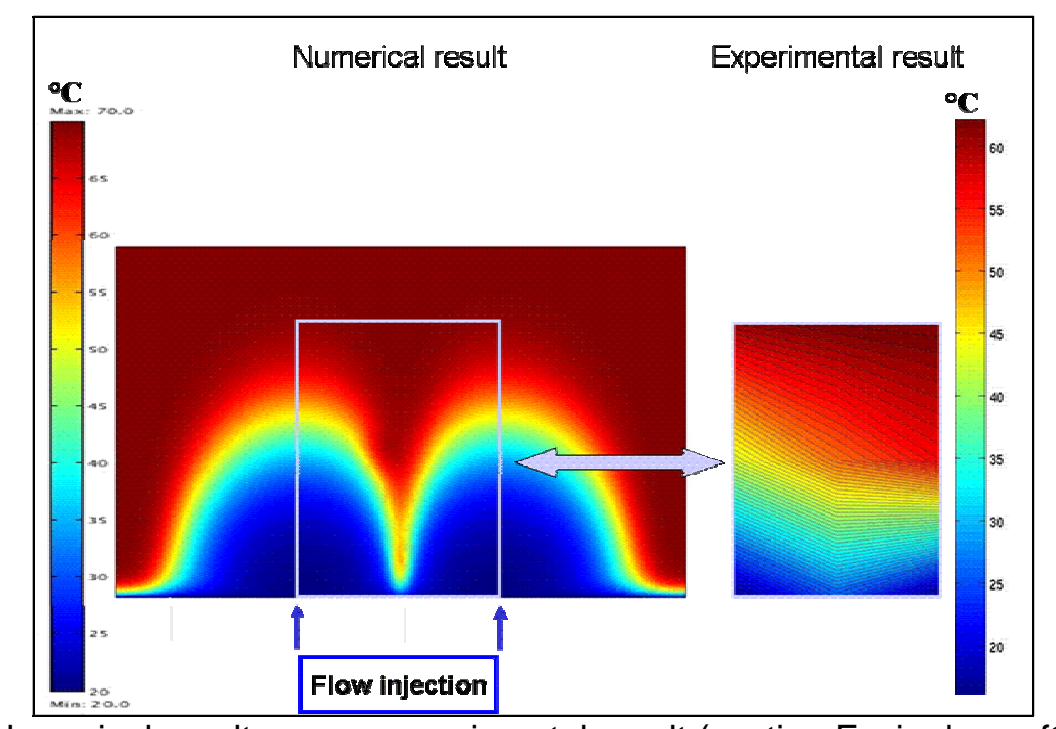

Figure 6. Numerical result versus experimental result (section F, six days after loading)

In a further objective of becoming an effective and quantitative tool for diagnosis and prediction, the numerical model will have to take into account:

- The temporal evolution of some parameters such as permeability and gas dispersion, due to the pore-scale evolution of the composting material,

- Local transfer phenomena (thermal convection, molecular diffusion).

The ways for the optimization of the composting process that have been proposed on the basis of the experimental result will have to be tested with the final composting numerical model.

\section{CONCLUSION}

The proposed experimental method of data acquisition and monitoring of heat flow in an industrial composting process has been validated. With this method, temperature fields obtained in a few transverse and longitudinal sections are already an essential tool to diagnose the process and to understand chemical reaction phenomena. Temperature fields in the transverse and longitudinal sections allow visualizing the temporal evolution of heat transfer in the compost. Heat transfer affects, throughout the composting cycle, the distribution of temperature and moisture in the waste, which are essential conditions for microbial activity. Obtaining temperature fields is then a first step towards a more sophisticated understanding of couplings between the microbiological phenomena which govern composting and heat and mass transfers. The study of the obtained data has also 
helped to propose future process optimizations. These proposals have to be supported by a more comprehensive numerical study in order to evaluate their impacts on the process.

\section{ACKNOWLEDGMENTS}

This work takes part in a larger project, entitled ESPACE and partially financed by the ANR (French national research agency), that is currently being carried out by Cemagref, SuezEnvironment and IMFT (French public research centre on fluid mechanics).

\section{REFERENCES}

Fernandes L., Zhan W., Patni N.K. and Jui P.Y., (1994), Temperature distribution and variation in passively aerated static compost piles, Bioresource Technology, 48, 257-263.

Haug R., (1993), The practical handbook of compost engineering. Lewis Publishers, Boca Raton, Florida.

Kulcu R. and Yaldiz O., (2008), Effects of air flow directions on composting process temperature profile. Waste Management, 28, 1766-1772.

NRAES-Northeast Regional Agricultural Engineering Service, (1992), On-Farm Composting Handboo, R. Rynk. Publication NRAES-S4, USA.

Richard T., (2004), Fundamentals parameters of aerobic solid-state bioconversion processes. Lens P, Hamelers B, Hoitink H,Bidlingmaier W. Resource Recovery and Reuse in Organic Solid Waste Management. IWA Publishing, London.

Tiquia S.M., Tam N.F.Y., and Hodgkiss I.J., (1996a), Microbial activities during composting of spent pig-manure sawdust litter at different moisture content, Bioresource. Technology, 55, 201-206.

Tiquia S.M., Tam N.F.Y. and Hodgkiss I.J., (1996b), Effects of compostingon phytotoxicity of spent pig-manure sawdust litter, Environ. Pollut., 93, 249-56.

Tiquia S.M., Tam N.F.Y. and Hodgkiss I.J., (1997), Effects of bacterial inoculum and moisture adjustement on composting of pig manure, Environ. Pollut., 96, 161-71.

Tremier A., De Guardia A., Massiani C. and Martel J.L., (2005a), Influence of the airflow rate on heat and mass transfers during sewage sludge and bulking agent composting, Environmental Technology, 26, 1137-1149.

Tremier A., De Guardia A., Massiani C., Paul E. and Martel J.L., (2005b), A respirometric method for characterising the organic comosition and biodegradation kinetics and the temperature influence on the biodegradation kinetics, for a mixture of sludge and bulking agent to be cocomposed, Bioresource Technology, 96, 169-180. 